\title{
CRESCIMENTO, PARTIÇÃo DE BIOMASSA E FOTOSSÍNTESE EM PLANTAS JOVENS DE Genipa spruceana SUBMETIDAS AO ALAGAMENTO
}

\author{
José Francisco de Carvalho Gonçalves ${ }^{1}$, Emanuelle Gurgel de Freitas Melo², Marciel José Ferreira ${ }^{3}$, \\ Carlos Eduardo Moura da Silva ${ }^{4}$, Iane Barroncas Gomes ${ }^{5}$
}

(recebido: 27 de maio de 2011; aceito: 25 de janeiro de 2013)

\begin{abstract}
RESUMO: Genipa spruceana Steyerm (Rubiaceae) é uma espécie frequentemente encontrada em ambientes alagados na Amazônia central. Neste estudo, objetivou-se elucidar possíveis estratégias adaptativas que permitem a espécie ocupar ambientes sujeitos ao alagamento, visando ao potencial da espécie para a restauração de áreas alagadas. No sentido de atingir tais objetivos foram investigadas características de crescimento, número de folhas, expansão foliar, acúmulo de biomassa, assimilação de carbono e condutância estomática de plantas jovens de G. spruceana submetidas aos tratamentos: 1- controle (SA-sem alagamento), 2- plantas parcialmente alagadas (PA) e 3- plantas totalmente alagadas (TA) durante 90 dias. Os tratamentos de alagamento PA e TA induziram menores incrementos em todas as variáveis de crescimento em altura e diâmetro quando comparados ao tratamento controle. Com o aumento do nível de inundação, a alocação de biomassa para a folha diminuiu até a completa abscisão foliar em TA, enquanto aumentou a massa caulinar. No tratamento PA, foi observada redução nas taxas de assimilação de carbono da ordem de $58 \%$ e $64 \%$ aos 60 e 90 dias, respectivamente, e de $96 \%$ aos 60 dias no tratamento TA. Entretanto, ao final do experimento todos os tratamentos apresentaram $100 \%$ de sobrevivência. Os resultados obtidos indicam que a perda de folhas e o ganho de biomassa no caule podem ser estratégias para atenuar o efeito prejudicial do alagamento. Por outro lado, as máximas taxas de sobrevivência sugerem que $G$. spruceana exibe alto potencial para estabelecimento em ambientes freqüentemente alagados.
\end{abstract}

Palavras-chave: Tolerância ao alagamento, assimilação de carbono, espécie arbórea tropical, rubiaceae.

\section{GROWTH, BIOMASS PARTITIONING AND PHOTOSYNTHESIS OF YOUNG PLANTS OF Genipa spruceana SUBJECTED TO FLOODING}

\begin{abstract}
Genipa spruceana Steyerm (Rubiaceae) is a species often found in flooded environments in the central Amazonia. The objective of this study was elucidate possible adaptive strategies that enable this species to occupy environments under flooding, targeting the potential of the species for restoration of floodplains. In order to achieve these objectives growth traits, number of leaves, leaf expansion, biomass production, carbon assimilation and stomatal conductance were investigated in G. spruceana seedlings subjected to treatments: 1- Non flooded plants (control-SA), 2- partially flooded (PA) and 3-completely flooded (TA) up to 90 days. Flooded treatments (PA and TA) induced smaller increments in all variables of height and diameter growth when compared to the control treatment. With increase of flooding, biomass allocation to leaves decreased until complete leaf abscission in TA, while increased in the stem. In PA treatment was observed reduction in C assimilation rates of $58 \%$ and $64 \%$ after 60 and 90 days, respectively, and 96\% after 60 days in TA treatment. However, in the end of the experiment all treatments presented $100 \%$ of survival. Our results indicate that the loss of leaves and gain of the stem biomass can be protective strategy to alleviate the harmful effects of the flooding. On the other hand, the maxim survival rates suggest that $G$. spruceana exhibit high potential for establishment in frequently flooded areas.
\end{abstract}

Key words: Flood tolerance, carbon assimilation, Rubiaceae, tropical tree species.

\section{INTRODUÇÃO}

As florestas sazonalmente inundadas e as áreas de depleção marginal dos reservatórios das hidrelétricas desempenham papel importante na dinâmica ambiental dos ecossistemas alagados do bioma amazônico, contribuindo para a conservação do solo, da qualidade da água, da flora e fauna aquática da região (FORSBERG et al.,

\footnotetext{
${ }^{1}$ Engenheiro Agrônomo, Pesquisador Doutor em Fisiologia Vegetal - Instituto Nacional de Pesquisas da Amazônia/INPA - Cx. P. 478 - 69011-970 Manaus, AM, Brasil - jfc@inpa.gov.br

${ }^{2}$ Engenheira Florestal, Mestra em Ciências de Florestas Tropicais - Instituto Nacional de Pesquisas da Amazônia/INPA - Cx. P. 478 - 69011-970 Manaus, AM, Brasil - emanuelegurgel@hotmail.com

${ }^{3}$ Engenheiro Florestal, Professor Doutor em Ciências de Florestas Tropicais - Universidade Federal do Amazonas - 69077-000 - Manaus, AM, Brasil-marciel@inpa.gov.br

${ }^{4}$ Engenheiro Florestal, Doutor em Ciências de Florestas Tropicais - Instituto Nacional de Pesquisas da Amazônia/INPA - Cx. P. 478 - 69011-970 Manaus, AM, Brasil - cems@inpa.gov.br

${ }^{5}$ Engenheira Florestal, Mestrando em Ciências de Florestas Tropicais - Instituto Nacional de Pesquisas da Amazônia/INPA - Cx. P. 478 - 69011970 - Manaus, AM, Brasil - iane.gomes@inpa.gov.br
}

Cerne, Lavras, v. 19, n. 2, p. 193-200, abr./jun. 2013 
1993; JUNK, 1997). Essas áreas, podem ainda exercer influência significativa nas condições climáticas regionais com potenciais implicações globais, uma vez que têm sido apontadas como relevante fonte de metano $\left(\mathrm{CH}_{4}\right)$ e outros gases do efeito estufa (PUEYO; FEARNSIDE, 2011).

Do ponto de vista da ecofisiologia vegetal, em ambientes sujeitos ao alagamento, a diminuição na concentração de oxigênio torna-se evidente, de maneira mais explicita naqueles órgãos da planta que são quase sempre permanentemente alagados e exibem elevadas demandas metabólicas, como, por exemplo, as raízes. Mesmo assim, algumas espécies arbóreas, a partir de suas frequências e ocorrências registradas em áreas alagadas, sinalizam potencial para tolerarem a baixa concentração de oxigênio, típica das áreas alagadas e, por conseguinte, exibem características adequadas para revegetar/restaurar ambientes submetidos a pulsos de alagamentos. Dentre essas espécies, destacam-se as do gênero Genipa (MIELKE et al., 2003). Espécies tolerantes à deficiência de oxigênio normalmente desenvolvem várias alterações morfológicas, funcionais e metabólicas, sendo as mais evidentes a expansão foliar; senescência, indução de abscisão foliar; diminuição nas trocas gasosas, ativação do metabolismo anaeróbico, redução do acúmulo de biomassa da raiz, folhas e hastes; hipertrofia de lenticelas e caules, formação de raízes adventícias, aerênquimas e pneumatóforos (FERREIRA et al., 2007; MEDINA et al., 2009; MIELKE et al., 2003, 2005; OLIVEIRA; JOLY, 2010; PAROLIN, 2003, 2009; ZANANDREA et al., 2010).

Na Amazônia, em particular na área de abrangência da represa hidrelétrica de Balbina, a espécie Genipa spruceana é bastante frequente, despertando interesse em investigar os fatores que alteram seu desempenho biométrico e funcional em condições de alagamento.

Genipa spruceana Steyerm (Rubiaceae) é uma espécie do tipo heliófita, semidecídua e seletiva higrófita, possui porte médio podendo alcançar $12 \mathrm{~m}$ de altura (REVILLA, 2002). Diante desses pressupostos, no presente trabalho, objetivou-se investigar possíveis estratégias adaptativas, que levam a alterações quanto ao crescimento e as características fotossintéticas, desenvolvidas por plantas jovens de Genipa spruceana Steyerm sob condições controladas de alagamento, no intuito de verificar se a mesma exibe crescimento, desempenho de ganho ou perda de biomassa e sobrevivência que sejam compatíveis com a tolerância ao estresse, quando exposta ou não ao alagamento.

Cerne, Lavras, v. 19, n. 2, p. 193-200, abr./jun. 2013

\section{MATERIAL E MÉTODOS}

\subsection{Local do estudo e instalação do experimento}

O experimento foi conduzido na área experimental do Instituto Nacional de Pesquisas da Amazônia (MCTI-

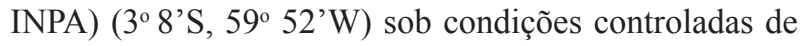
alagamento. Para tanto, plantas jovens $( \pm 35,0 \mathrm{~cm}$ de altura e em bom estado fitossanitário) foram coletadas no entorno da Reserva Biológica do Uatumã, distante $180 \mathrm{~km}$ de Manaus-AM, e transferidas para vasos de $5 \mathrm{~kg}$, contendo mistura de areia e terriço (1:1), peneirados em peneira de $0,5 \mathrm{~cm}$. Após aclimatação (durante um mês em sol pleno), as plantas foram submetidas aos tratamentos: 1controle (SA - sem alagamento), 2- plantas parcialmente alagadas (PA) e 3-plantas totalmente alagadas (TA). Cada tratamento continha cinco repetições representadas por tanques de água com capacidade para 1000 litros (L), sendo cinco plantas por tanque (pseudo-repetições). Portanto, o número total de indivíduos amostrados no experimento foi igual a 75 plantas. $\mathrm{O}$ espaçamento entre as plantas envasadas foi de aproximadamente $40 \mathrm{~cm}$ e as mesmas foram submetidas à inundação durante um período de 90 dias. A água dos tanques foi trocada a cada três dias, a fim de evitar potenciais interferências advindas de mudanças nas características físicas e químicas da água que viessem a mascarar o efeito da disponibilidade de oxigênio, além de promoverem alterações oxido redutivas inerentes a presença dos solos envasados dentro dos tanques.

\subsection{Análises de crescimento e sobrevivência}

O estabelecimento das plantas jovens de $G$. spruceana foi analisado utilizando-se o método não destrutivo, a partir da determinação das taxas de crescimento relativo e absoluto em altura, diâmetro do coleto e número de folhas. A determinação das variáveis de crescimento foi realizada em dois momentos, sendo o primeiro na implantação do experimento e o segundo aos 90 dias de experimentação, em dois indivíduos em cada repetição e cinco repetições por tratamento. $\mathrm{O}$ crescimento relativo em altura (CR-a), diâmetro do coleto (CR-d) e o índice de ganho foliar (IGF) foram calculados conforme Bugbee (1996) e Davanso et al. (2002). O crescimento absoluto em altura (CA-a), em diâmetro do coleto (CA-d) e ganho foliar (GF), foram determinados pela diferença entre as medidas realizadas no início e no término do experimento. 
A porcentagem de sobrevivência das plantas foi determinada a partir da razão entre o total de plantas usadas na implantação e o número de plantas vivas após 90 dias do início do experimento.

Ao final do experimento, indivíduos de cada tratamento foram seccionados em raiz, caule e folhas, colocados separadamente dentro de sacos de papel com massa conhecida e após, essas partes permanecerem em estufa a $60^{\circ} \mathrm{C} \pm 3^{\circ} \mathrm{C}$ durante 72 horas, depois foram novamente pesados em balança analítica (Shimadzu, modelo AY220) para obtenção da biomassa seca.

\subsection{Determinação das trocas gasosas}

As variáveis de trocas gasosas foram obtidas por meio de um analisador de gás a infravermelho (IRGA) portátil, de sistema aberto, modelo LI-6400. Os dados foram coletados entre 8:00 e 12:00 horas, em folhas completamente expandidas, em adequado estado fitossanitário e localizadas no terço médio das plantas. As variáveis de trocas gasosas foram determinadas com a câmara foliar ajustada para a concentração de $\mathrm{CO}_{2}$, temperatura e vapor de $\mathrm{H}_{2} \mathrm{O}$ em torno de $385 \pm 10 \mu \mathrm{mol}$ $\mathrm{mol}^{-1}, 31 \pm 1^{\circ} \mathrm{C}$ e $21 \pm 1 \mathrm{mmol} \mathrm{mol}^{-1}$, respectivamente. Os dados da curva de resposta fotossintética em relação à intensidade luminosa foram obtidos à densidade de fluxo de fótons (PPDF) entre 0 e $2000 \mu \mathrm{mol} \mathrm{m}^{-2} \mathrm{~s}^{-1}$, medidos em escala decrescente para onze níveis de irradiância (2000, $1500,1000,750,500,250,100,75,50,25 \mathrm{e} 0 \mu \mathrm{mol} \mathrm{m}^{-2} \mathrm{~s}^{-1}$ ). O modelo da equação exponencial foi usado para ajustar a curva de resposta fotossintética a intensidade luminosa para cada planta (IQBAL et al., 1997). Os valores de $A_{\max }$ e $\alpha$ foram estimados utilizando-se a regressão exponencial por meio do programa Statistica 6.0 versão Windows (StatSoft, Inc., Tulsa, USA). A irradiância de compensação de luz $\left(\mathrm{I}_{\mathrm{c}}\right.$; PPDF no qual $\left.\mathrm{A}=0\right)$ foi calculada pela fórmula $\mathrm{I}_{\mathrm{c}}=\mathrm{R}_{\mathrm{d} /} \alpha$ e a irradiância de saturação de luz (I ; PPDF no qual $\mathrm{A}=90 \% A_{\max }$ ).

\subsection{Delineamento experimental e análises estatísticas}

O delineamento experimental utilizado foi o inteiramente casualizado, com três tratamentos: 1controle (SA - sem alagamento), 2- plantas parcialmente alagadas (PA) e 3- plantas totalmente alagadas (TA). Cada tratamento foi composto por cinco repetições (tanques) e cada repetição constituída por cinco indivíduos. Os resultados obtidos foram submetidos ao teste de normalidade de Lilliefors e, em seguida, submetidos à análise de variância (ANOVA), sendo, posteriormente, comparados pelo teste de Tukey $(p<0,05)$. A escolha feita pela análise de variância, seguida do teste de Tukey, mesmo para dados com características quantitativas (tempo de alagamento), deve-se ao fato dos tratamentos de alagamento (SA, PA e TA) estarem combinados ao fator tempo de exposição ao estresse, assumindo-se assim caráter qualitativo. O programa utilizado para a realização das análises estatísticas foi o Statistica 6.0 (STATSOFT, 2003).

\section{RESULTADOS E DISCUSSÃO}

Os valores médios de crescimento relativo em diâmetro do coleto (CR-d) foram $0,0035 \mathrm{~mm} \mathrm{~mm}^{-1} \mathrm{dia}^{-1}$ na condição sem alagamento (SA), $0,0023 \mathrm{~mm} \mathrm{~mm}^{-1}$ dia $^{-1}$ na condição parcialmente alagado (PA) e $0,0013 \mathrm{~mm}$ $\mathrm{mm}^{-1}$ dia $^{-1}$ na condição totalmente alagado (TA). Quanto ao crescimento relativo em altura (CR-a), os tratamentos PA e TA exibiram valores $36 \%$ e $67 \%$, respectivamente, menores que o tratamento SA. Por último, os valores de índice de ganho foliar (IGF) foram 4,00 (SA), -3,00 (PA) e $-8,89$ (TA). As taxas de crescimento absoluto em diâmetro do coleto (CA-d) variaram entre $0,69 \mathrm{~mm}$ (TA) e $2,08 \mathrm{~mm}$ (SA). Quanto ao crescimento absoluto em altura (CA-a), os tratamentos PA e TA apresentaram valores $46 \%$ e $81 \%$, respectivamente, menores que o tratamento SA. Por sua vez, os valores de ganho foliar (GF) variaram entre $-4,00$ (TA) e 7,80 (SA), sendo que os tratamentos PA e TA exibiram ganhos $68 \%$ e $151 \%$ respectivamente menores que SA (Figura 1).

Plântulas de Genipa americana submetidas ao alagamento, durante 63 dias, apresentaram diminuição de $30 \%$ tanto em altura como em número de folhas, enquanto diferenças para o diâmetro do coleto não foram observadas (MIELKE et al., 2003). Todavia, plantas de carnaúba não apresentaram aumento na altura da parte aérea (ARRUDA; CALBO, 2004). Do mesmo modo, plântulas de Annona glabra submetidas ao alagamento durante 56 dias, não apresentaram diferenças em altura e número de folhas, contudo houve aumento de $14 \%$ do diâmetro do coleto quando submetidas à submersão (MIELKE et al., 2005). Calophyllum brasiliense também exibiu como sintomas de estresse ao alagamento redução do crescimento em altura e emissão de novas folhas, embora o crescimento em diâmetro tenha sido superior nas plantas submetidas ao alagamento (OLIVEIRA; JOLY, 2010). Por outro lado, em Erythrina speciosa o tratamento de alagamento não induziu diferenças no crescimento em altura em relação ao controle (não alagado), porém, entre o $14^{\circ}$ e $40^{\circ}$ dia de imposição

Cerne, Lavras, v. 19, n. 2, p. 193-200, abr./jun. 2013 




Figura 1 - Box plot das taxas de crescimento relativo (a) em diâmetro do coleto, (b) altura e (c) índice de ganho foliar; e crescimento absoluto (d) em diâmetro do coleto, (e) altura e (f) ganho foliar em plantas jovens de G. spruceana submetidas a 90 dias de alagamento. Linha contínua no interior do box - mediana, linha pontilhada no interior do box - média, $25^{\circ}$ e $75^{\circ}$ percentis, com barras de erro exibindo valores do $10^{\circ}$ e $90^{\circ}$ percentis. $\mathrm{SA}=$ Sem alagamento; $\mathrm{PA}$ $=$ Parcialmente alagado e TA $=$ Totalmente alagado. Médias seguidas pelas mesmas letras não diferem entre si pelo teste de Tukey $(P<0,05)$.

Figure 1 - Box plot of the relative growth rate in (a) diameter at collar height, (b) height and (c) leaf gain index; and absolute growth in (d) diameter at the stem base, (e) height and (f) leaf gain in young plants of G. spruceana subjected to 90 days of flooding. Continuous line inside the box-median, dashed line inside the box-mean, 25th and 75th percentile values, with error bars showed 10th and 90th percentile values. $S A=$ no flooding; $P A=$ partially flooded and $T A=$ completely flooded. Means followed by the same letters do not differ according to the Tukey test $(P<0.05)$.

Cerne, Lavras, v. 19, n. 2, p. 193-200, abr./jun. 2013 do tratamento, plantas alagadas exibiram crescimento em diâmetro 20\% maior que o controle (MEDINA et al., 2009). Em síntese, as respostas são diversas e, sempre que há crescimento, os incrementos não são de grande escala, sugerindo que o gasto energético utilizado nos mecanismos de tolerância pode representar elevado custo para a planta. As análises feitas com os dados coletados no final do experimento também demonstraram mudanças no crescimento de $G$. spruceana. No tratamento controle (SA), a biomassa de folha correspondeu a $22 \%$ da biomassa total, enquanto a biomassa de caule $32 \%$ e a biomassa de raiz 46\% (Figura 2). De acordo com o aumento do nível de inundação, a alocação de biomassa para a folha diminuiu até a completa abscisão foliar observada no tratamento TA, enquanto a biomassa para o caule aumentou. Algumas espécies podem exibir como estratégia de tolerância ao alagamento total, indução da senescência foliar como resposta a redução de assimilados na folha, culminando com a abscisão foliar (MAURENZA et al., 2012). Por sua vez, a biomassa de raiz em TA representou $62 \%$ da biomassa total da planta. $\mathrm{O}$ efeito do alagamento sobre o acúmulo e alocação de biomassa de plantas de Genipa americana também foi investigado durante 63 dias e os resultados indicaram

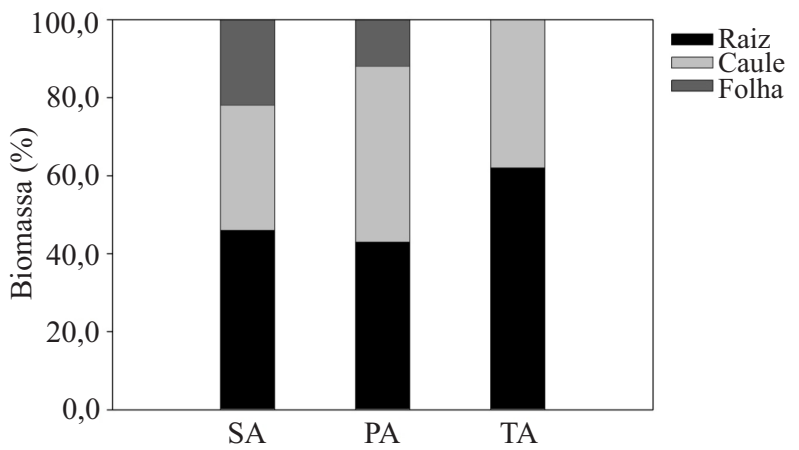

Figura 2 - Porcentagem de biomassa de raiz, caule e folha em plantas jovens de $G$. spruceana. $\mathrm{SA}=$ Sem alagamento, $\mathrm{PA}=$ Parcialmente alagado e TA $=$ Totalmente alagado, aos 90 dias de alagamento. *Os indivíduos do tratamento totalmente alagado (TA) apresentavam completa abscisão foliar aos 90 dias de experimento.

Figure 2 - Percentage of root biomass, stem and leaf in Young plants of $G$. spruceana. $S A=$ no flooding; $P A=$ partially flooded and TA = completely flooded, after 90 days of flooding. *Individuals belonging to completely flooded treatment (TA) showed complete leaf abscission at 90 days of experiment. 
que plântulas submetidas ao alagamento apresentaram diminuição de $59 \%$ na biomassa de raízes e de $40 \%$ na biomassa de folhas (MIELKE et al., 2003). Por outro lado, plântulas de Annona glabra submetidas ao alagamento durante 56 dias apresentaram incrementos de $52,5 \%$ e $34 \%$ na biomassa de raízes e caule, respectivamente, contudo não houve diferenças para biomassa de folhas (MIELKE et al., 2005). Erythrina speciosa alocou maior fração de biomassa para a parte aérea aos 28 e 45 dias após imposição dos tratamentos de alagamento quando comparado à condição não alagada (MEDINA et al., 2009). O alagamento também induziu menores valores de produção de matéria seca de raízes, caule e folhas em Pouteria glomerata e Calophyllum brasiliense, embora de acordo com o aumento do nível de inundação em P. glomerata, a biomassa fotoassimilada foi alocada preferencialmente para o caule (MAURENZA et al., 2009; OLIVEIRA; JOLY, 2010). Por último, em Eugenia uniflora o alagamento resultou valores $20 \%, 58 \%$ e $27 \%$ menores que o controle (não alagado) para a produção de matéria seca da parte aérea, radicular e total, respectivamente (MIELKE; SCHAFFER, 2010b).

Apesar das diferenças observadas nas variáveis de crescimento, todos os tratamentos apresentaram, aos 90 dias de experimento, $100 \%$ dos indivíduos vivos. Contudo, alterações morfológicas foram verificadas no tratamento PA que, aos 15 dias de alagamento apresentou formação de lenticelas em todos os indivíduos, ao passo que no tratamento TA ocorreu abscisão foliar a partir de 30 dias com perda total das folhas aos 60 dias. Resultados semelhantes na presente pesquisa, foram observados em Erythrina speciosa que também apresentou 100\% de sobrevivência dos indivíduos após 60 dias de imposição dos tratamentos de alagamento, com hipertrofia de lenticelas e formação de raízes adventícias e aerênquima.
Essas modificações morfológicas representam estratégias desenvolvidas por plantas submetidas ao estresse por alagamento e contribuem para a melhor aeração do sistema radicular (MEDINA et al., 2009). Por outro lado, o alagamento afetou a sobrevivência de Eugenia uniflora, causando redução de 10\% quando comparado ao tratamento controle (não alagado) (MIELKE; SCHAFFER, 2010b).

Os valores médios de fotossíntese máxima $\left(A_{\max }\right)$ variaram entre $0,36 \mu \mathrm{mol} \mathrm{m} \mathrm{m}^{-2} \mathrm{~s}^{-1}$ (TA-60 dias) e $10,65 \mu \mathrm{mol} \mathrm{m} \mathrm{m}^{-2} \mathrm{~s}^{-1}$ (SA-30 dias), sendo que no início do experimento (0 dias), plantas de G. spruceana apresentaram $A_{\max }$ de $9,46 \mu \mathrm{mol} \mathrm{m} \mathrm{m}^{-2} \mathrm{~s}^{-1}$. Contudo, com o decorrer do tempo de submissão das plantas ao alagamento, observaram-se reduções de $58 \%$ e $64 \%$ em PA aos 60 e 90 dias, respectivamente, e de $96 \%$ em TA aos 60 dias quando comparados ao controle (SA) (Tabela 1) (Figura 3). A condutância estomática (gs) variou entre $0,07 \mathrm{~mol} \mathrm{~m}^{-2} \mathrm{~s}^{-1}$ (PA e TA-60 dias) e $0,31 \mathrm{~mol} \mathrm{~m}^{-2}$ $\mathrm{s}^{-1}$ (SA-90 dias), havendo reduções de $43 \%$ (PA) e $67 \%$ (TA) aos 90 e 60 dias de alagamento, respectivamente. Por sua vez, o rendimento quântico $(\alpha)$ variou entre 0,04 e $0,06 \mathrm{~mol} \mathrm{CO}_{2} \mathrm{~mol}^{-1}$ quanta em todos os tratamentos ao longo do período de alagamento. Teoricamente, o valor máximo que $\alpha$ pode assumir é 0,125 , equivalente a 8 moles de fótons requeridos para reduzir $1 \mathrm{~mol} \mathrm{de} \mathrm{CO}_{2}$ na ausência de fotorrespiração (SINGSAAS et al., 2001). Assim, comparando os valores de $\alpha$ encontrados para $G$. spruceana, verificaram-se reduções entre $52 \%$ e $68 \%$ se comparados ao valor máximo teórico. Os valores de respiração foliar (Rd) variaram de $0,55-1,37 \mu \mathrm{mol} \mathrm{m}^{-2}$ $\mathrm{s}^{-1}$ (SA), $0,80-1,37 \mu \mathrm{mol} \mathrm{m} \mathrm{m}^{-2} \mathrm{~s}^{-1}$ (PA) e de $0,29-1,37$ $\mu \mathrm{mol} \mathrm{m} \mathrm{m}^{-2} \mathrm{~s}^{-1}$ (TA). Os baixos valores de $\alpha$ e de Rd foram determinantes para a redução dos valores de irradiância de compensação (Ic), que atingiram em TA aos 60 dias

Tabela 1 - Resultados da ANOVA (valores de F) para os principais efeitos e interações dos parâmetros: fotossíntese máxima (A ${ }_{\text {max }}$ ), condutância estomática (gs), irradiância de compensação (Ic), irradiância de saturação (Is), rendimento quântico $(\alpha)$ e respiração no escuro (Rd) em plantas jovens de G. spruceana submetidas a 90 dias de alagamento ( $\mathrm{n}=5)$. Graus de liberdade $(\mathrm{GL}){ }^{*}=\mathrm{P}<0,001$.

Table 1 - ANOVA results ( $F$ values) for the main effects and interactions of the parameters: Light-saturated photosynthetic rate $\left(A_{\max }\right)$, stomatal conductance $(g s)$, compensation irradiance (Ic), saturation irradiance (Is), quantum yield $(\alpha)$ and dark respiration (Rd) in young plants of $G$. spruceana subjected to 90 days of flooding $(n=5)$. Degree of freedom $(D F)$. $*=P<0.001$.

\begin{tabular}{lccccccc}
\hline Efeito & $\mathrm{GL}$ & $\mathrm{A}_{\max }$ & $\mathrm{gs}$ & $\mathrm{Ic}$ & $\mathrm{Is}$ & $\alpha$ & $\mathrm{Rd}$ \\
\hline Alagamento (A) & 2 & $40,72^{*}$ & $8,76^{*}$ & $17,97^{*}$ & $33,27^{*}$ & $10,3^{*}$ & $26,26^{*}$ \\
Período (P) & 4 & $36,1^{*}$ & $12,63^{*}$ & $32,25^{*}$ & $10,43^{*}$ & $25,72^{*}$ & $82,66^{*}$ \\
Interação A x P & 8 & $9,58^{*}$ & $5,9^{*}$ & $6,31^{*}$ & $7,52^{*}$ & $15,94^{*}$ & $16,05^{*}$ \\
\hline
\end{tabular}

Cerne, Lavras, v. 19, n. 2, p. 193-200, abr./jun. 2013 


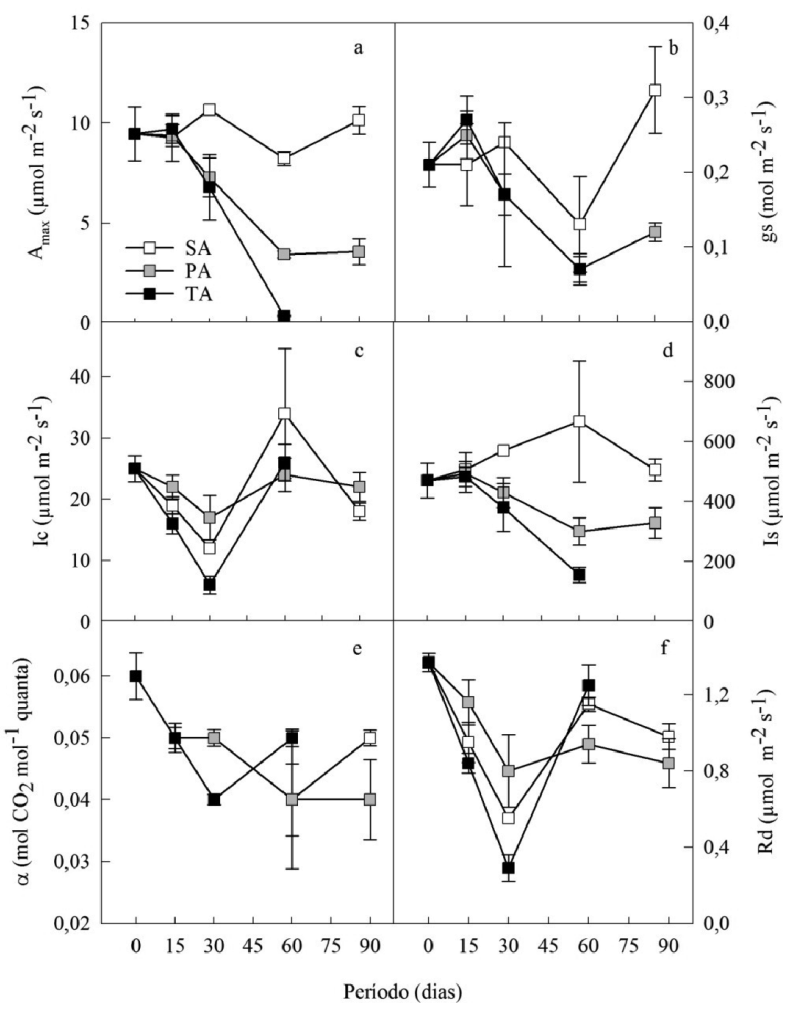

Figura 3 - Fotossíntese máxima $\left(A_{\max }\right)$ (a), condutância estomática (gs) (b), irradiância de compensação (Ic) (c), irradiância de saturação (Is) (d), rendimento quântico $(\alpha)$ (e) e respiração no escuro (Rd) (f), em plantas jovens de G. spruceana submetidas a 90 dias de alagamento. Os valores são médias e as linhas verticais indicam os erros-padrão. *Os indivíduos do tratamento totalmente alagado (TA) apresentavam completa abscisão foliar aos 90 dias de experimento.

Figure 3 - Light-saturated photosynthetic rate $\left(A_{\max }\right)(a)$, stomatal conductance (gs) (b), compensation irradiance (Ic) (c), saturation irradiance (Is) (d), quantum yield ( $\alpha$ ) (e) and dark respiration $(R d)(f)$, in young plants of $G$. spruceana subjected to 90 days of flooding. Values are means and vertical lines indicate the standard error. *Individuals belonging to completely flooded treatment (TA) showed complete leaf abscission at 90 days of experiment.

de alagamento $6 \mu \mathrm{mol} \mathrm{m} \mathrm{m}^{-2} \mathrm{~s}^{-1}$; enquanto os valores de irradiância de saturação variaram entre 470 e $667 \mu \mathrm{mol}$ $\mathrm{m}^{-2} \mathrm{~s}^{-1}$ (SA), $300-494 \mu \mathrm{mol} \mathrm{m} \mathrm{m}^{-2} \mathrm{~s}^{-1}$ (PA) e 155 - 482 $\mu \mathrm{mol} \mathrm{m} \mathrm{m}^{-2} \mathrm{~s}^{-1}$ (TA).

Espécies pioneiras típicas de ambientes de várzea Amazônica (Cecropia latiloba e Senna reticulata) mostraram redução de $10 \%$ nas taxas de fotossíntese máxima, durante o período de alagamento, ao passo que espécies não-pioneiras (Nectandra amazonum, Crateva benthami, Tabebuia barbata e Vitex cymosa) experimentaram reduções entre $20 \%$ e $50 \%$, quando comparado com o período seco. Por sua vez, o rendimento quântico exibiu valores entre $20 \%$ e $50 \%$ menores nas espécies não-pioneiras no período alagado e entre $2 \%$ e $5 \%$ superiores nas espécies pioneiras (PAROLIN, 2000; PAROLIN et al., 2001). Genipa americana submetida ao alagamento apresentou redução de $51 \%$ em fotossíntese máxima e $27 \%$ em respiração no escuro, embora diferenças associadas ao rendimento quântico não tenham sido observadas neste experimento (MIELKE et al., 2003). Investigações a respeito das reações fisiológicas de Pouteria glomerata e Laetia corymbulosa em áreas inundáveis da Amazônia, observaram que a atividade fotossintética de $P$. glomerata permaneceu constante entre 8 e $12 \mu \mathrm{mol}$ $\mathrm{m}^{-2} \mathrm{~s}^{-1}$, enquanto que em $L$. corymbulosa foi maior na fase terrestre $\left(13 \mu \mathrm{mol} \mathrm{m} \mathrm{m}^{-2} \mathrm{~s}^{-1}\right)$, sendo após seis meses de alagamento acentuadamente reduzida para $3 \mu \mathrm{mol} \mathrm{m} \mathrm{m}^{-2}$ $\mathrm{s}^{-1}$ (ARMBRÜSTER et al., 2004). Do mesmo modo, o alagamento induziu em Genipa americana diminuição de $64 \%$ das taxas fotossintéticas, contudo diferenças nas taxas de respiração no escuro entre plantas alagadas e não alagadas não foram observadas (LAVINSKY et al., 2007). Reduções nas taxas de fotossíntese e condutância estomática têm sido verificadas em grande número de espécies arbóreas submetidas ao alagamento (MAURENZA et al., 2009; MEDINA et al., 2009; MIELKE; SCHAFFER, 2010a, 2010b). Alterações nas taxas de assimilação de carbono em plantas submetidas ao alagamento podem estar associadas tanto ao fechamento estomático quanto a limitações não estomáticas, como modificações na atividade de enzimas envolvidas no processo de carboxilação e degradação de pigmentos (MIELKE; SCHAFFER, 2010a). No presente experimento, limitações estomáticas pareceram exercer influência preponderante sobre as taxas de assimilação de carbono das plantas submetidas ao alagamento. $\mathrm{O}$ fato é que reduções nas taxas de condutância estomática com o decorrer do período de alagamento foram muito mais significativas se comparadas às reduções no rendimento quântico (Figura 3).

Em resumo, os resultados obtidos no presente estudo indicam que o efeito depletivo do alagamento sobre as taxas de assimilação de carbono em G. spruceana é inevitável. Ao mesmo tempo, a ativação de mecanismos de tolerância pode estar relacionada ao tempo de exposição ao estresse, uma vez que no tratamento PA houve diminuição gradual das taxas de fotossíntese máxima que tenderam a

Cerne, Lavras, v. 19, n. 2, p. 193-200, abr./jun. 2013 
se estabilizar entre 60 e 90 dias de alagamento. Todavia, mesmo apresentando menores taxas de crescimento e redução na assimilação de carbono quando submetida ao alagamento, a espécie G. spruceana demonstrou capacidade para sobreviver e acumular biomassa. A capacidade para crescer em ambientes sujeitos a pulsos de alagamento, como às margens da hidrelétrica de Balbina, sugere que $G$. spruceana exibe características ecofisiológicas adequadas para compor atividades destinadas à restauração de áreas periodicamente alagadas como, por exemplo, programas de revegetação florestal nas margens de usinas hidroelétricas.

\section{AGRADECIMENTOS}

Ao Instituto Nacional de Pesquisas da Amazônia (MCTI-INPA) e ao Conselho Nacional de Desenvolvimento Científico e Tecnológico (CNPq), pelo auxílio pesquisa ao projeto Ref. 553316/2005-2, pelo financiamento e concessão das bolsas (IC, DTI e PQ).

\section{REFERÊNCIAS}

ARMBRÜSTER, N.; MÜLLER, E.; PAROLIN, P. Contrasting responses of two Amazonian floodplain trees to hydrological changes. Ecotropica, Frankfurt, v. 10, n. 2, p. 73-84, Apr. 2004.

ARRUDA, G. M. T.; CALBO, M. E. R. Efeitos da inundação no crescimento, trocas gasosas e porosidade radicular da carnaúba (Copernicia prunifera (Mill.) H. E. Moore). Acta Botanica Brasilica, São Paulo, v. 18, n. 2, p. 219-224, abr. 2004.

BUGBEE, B. G. Growth, analysis and yield components. In: SALISBURY, F. B. (Ed.). Units, symbols, and terminology for plant physiology. Oxford: Oxford University, 1996. p. 115-119.

DAVANSO, V. M.; SOUZA, L. A.; MEDRI, M. E.; PIMENTA, J. A.; BIANCHINI, E. Photosynthesis, growth and development of Tabebuia avellanedae Lor. Ex Griseb. (Bignoniaceae) in flooded soil. Brazilian Archives of Biology and Technology, Curitiba, v. 45 , n. 3, p. 375-384, Sept. 2002.

FERREIRA, C. S.; PIEDADE, M. T. F.; JUNK, W. J.; PAROLIN, P. Floodplain and upland populations of Amazonian Himatanthus sucuuba: effects of flooding on germination, seedling and mortality. Environment and Experimental Botany, Oxford, v. 60 , p. 477-483, July 2007.

FORSBERG, C. A.; ARAÚJO-LIMA, R. M.; MARTINELLI, L. A.; VICTORIA, R. L.; BONASSI, J. A. Autotrophic carbon sources for fish of central Amazon. Ecology, New York, v. 74, p. 643-652, Apr. 1993.

IQBAL, R. M.; RAO, A. R.; RASUL, E.; WAHID, A. Mathematical model and response functions in photosynthesis: an exponential model. In: PESSARAKLI, M. (Ed.). Handbook of photosynthesis. New York: M. Dekker, 1997. p. 803-810.

JUNK, W. J. The Central Amazon floodplain: ecology of pulsing system. Berlin: Springer Verlag, 1997. 525 p.

LAVINSKY, A. O.; SANT'ANA, C. S.; MIELKE, M. S.; ALMEIDA, A. F.; GOMES, F. P.; FRANÇA, S.; SILVA, D. C. Effects of light availability and soil flooding on growth and photosynthetic characteristics of Genipa americana L. seedlings. New Forests, Dordrecht, v. 34, n. 1, p. 41-50, July 2007.

MAURENZA, D.; MARENCO, R. A.; PAROLIN, P.; PIEDADE, M. T. F. Physiological responses to flooding and light in two tree species native to the Amazonian floodplains. Aquatic Botany, Amsterdam, v. 96, n. 1, p. 7-13, Jan. 2012.

MAURENZA, D.; MARENCO, R. A.; PIEDADE, M. T. F. Efeito da inundação de longa duração sob o crescimento de Pouteria glomerata (Sapotaceae), uma arbórea da várzea da Amazônia Central. Acta Amazonica, Manaus, v. 39, n. 3, p. 519-526, Sept. 2009.

MEDINA, C. L.; SANCHES, M. C.; TUCCI, M. L. S.; SOUSA, C. A. F.; CUZZUOL, G. R. F.; JOLY, C. A. Erythrina speciosa (Leguminosae-Papilionoideae) under soil water saturation: morphophysiological and growth responses. Annals of Botany, Oxford, v. 104, p. 671-680, Sept. 2009.

MIELKE, M. S.; ALMEIDA, A. F.; GOMES, F. P.; AGUILAR, M. A. G.; MANGABEIRA, P. A. O. Leaf gas exchange, chlorophyll fluorescence and growth responses of Genipa americana seedlings to soil flooding. Environment and Experimental Botany, Oxford, v. 50, n. 3, p. 221-231, Dec. 2003.

MIELKE, M. S.; MATOS, E. M.; COUTO, V. B.; ALMEIDA, A. F.; GOMES, F. P.; MANGABEIRA, P. A. O. Some photosynthetic and growth responses of Annona glabra L. seedling to soil flooding. Acta Botanica Brasilica, São Paulo, v. 19, n. 4, p. 905-911, jul. 2005.

MIELKE, M. S.; SCHAFFER, B. Leaf gas exchange, chlorophyll fluorescence and pigment indexes of Eugenia uniflora L. in response to changes in light intensity and soil flooding. Tree Physiology, Oxford, v. 30, n. 1, p. 45-55, Jan. 2010a.

Cerne, Lavras, v. 19, n. 2, p. 193-200, abr./jun. 2013 
MIELKE, M. S.; SCHAFFER, B. Photosynthetic and growth responses of Eugenia uniflora L. seedlings to soil flooding and light intensity. Environment and Experimental Botany, Oxford, v. 68, n. 2, p. 113-121, Apr. 2010b.

OLIVEIRA, V. C.; JOLY, C. A. Flooding tolerance of Calophyllum brasiliense Camb. (Clusiaceae): morphological, physiological and growth responses. Trees, Berlin, v. 24, n. 1, p. 185-193, Feb. 2010.

PAROLIN, P. Fugitive and possessive establishment strategies in Amazonian floodplain pioneers. Flora, London, v. 198, n. 6, p. 436-443, 2003.

PAROLIN, P. Phenology and $\mathrm{CO}_{2}$-assimilation of trees in Central Amazonian floodplains. Journal of Tropical Ecology, New York, v. 16, n. 3, p. 465-473, Nov. 2000.

PAROLIN, P. Submerged in darkness: adaptations to prolonged submergence by woody species of the Amazonian floodplains. Annals of Botany, Oxford, v. 103, n. 2, p. 359376, Jan. 2009.
PAROLIN, P.; JUNK, W. J.; PIEDADE, M. T. F. Gas exchange of six tree species from Central Amazonian floodplains. Tropical Ecology, New Delhi, v. 42, n. 1, p. 15-24, 2001.

PUEYO, S.; FEARNSIDE, P. M. Emissões de gases de efeito estufa dos reservatórios de hidrelétricas: implicações de uma lei de potência. Oecologia Australis, Rio de Janeiro, v. 15, n. 2, p. 199-212, jul. 2011.

REVILLA, J. Plantas úteis da bacia amazônica. Manaus: SEBRAE-AM/INPA, 2002. 289 p.

SINGSAAS, E. L.; ORT, D. R.; DELUCIA, E. H. Variation in measured values of photosynthetic quantum yield in ecophysiological studies. Oecologia, Berlin, v. 128, p. 15-23, June 2001.

STATSOFT. Statistica. Version 6.0. Tulsa, 2003.

ZANANDREA, I.; ALVES, J. D.; DEUNER, S.; GOULART, P. F. P.; HENRIQUE, P. C.; SILVEIRA, N. M. Tolerance of Sesbania virgata plants to flooding. Australian Journal of Botany, Melbourne, v. 57, n. 8, p. 661-669, Feb. 2010.

Cerne, Lavras, v. 19, n. 2, p. 193-200, abr./jun. 2013 